\title{
Front Matter: Volume 6997
}

, "Front Matter: Volume 6997," Proc. SPIE 6997, Semiconductor Lasers and Laser Dynamics III, 699701 (6 June 2008); doi: 10.1117/12.803014

SPIE. Event: SPIE Photonics Europe, 2008, Strasbourg, France 


\title{
PROCEEDINGS OF SPIE
}

\section{Semiconductor Lasers \\ and Laser Dynamics III}

\author{
Krassimir P. Panajotov \\ Marc Sciamanna \\ Angel A. Valle \\ Rainer Michalzik \\ Editors
}

\section{7-9 April 2008}

Strasbourg, France

Sponsored by

SPIE Europe

Cosponsored by

Alsace International (France)

Conseil Général du Bas-Rhin (France)

Région Alsace (France)

Communauté Urbaine de Strasbourg (France)

Cooperating Organizations

AFOP_Association Française des Industries de l'Optique et de la Photonique (France)

EOS-European Optical Society (Germany) • EPIC—European Photonics Industry Consortium

(France) • ePIXnet (Belgium) • IOP—Institute of Physics (United Kingdom) • NEMO-Network of

Excellence on Micro-Optics (Belgium) • OLAS (Italy) • The OLLA Project (Germany) • OPERA 2015

(Belgium) • PhOREMOST (Ireland) • Photonics Knowledge Transfer Network (United Kingdom)

Photonics Cluster (United Kingdom) • Photonics4Life (Germany) • Photonics 21 (Germany)

RhenaPhotonics Alsace (France)

Published by

SPIE

Volume 6997 
The papers included in this volume were part of the technical conference cited on the cover and title page. Papers were selected and subject to review by the editors and conference program committee. Some conference presentations may not be available for publication. The papers published in these proceedings reflect the work and thoughts of the authors and are published herein as submitted. The publisher is not responsible for the validity of the information or for any outcomes resulting from reliance thereon.

Please use the following format to cite material from this book:

Author(s), "Title of Paper," in Semiconductor Lasers and Laser Dynamics III, edited by Krassimir P. Panajotov, Marc Sciamanna, Angel A. Valle, Rainer Michalzik, Proceedings of SPIE Vol. 6997 (SPIE, Bellingham, WA, 2008) Article CID Number.

ISSN 0277-786X

ISBN 9780819471956

Published by

SPIE

P.O. Box 10, Bellingham, Washington 98227-0010 USA

Telephone +1 3606763290 (Pacific Time) · Fax +1 3606471445

SPIE.org

Copyright (c) 2008, Society of Photo-Optical Instrumentation Engineers

Copying of material in this book for internal or personal use, or for the internal or personal use of specific clients, beyond the fair use provisions granted by the U.S. Copyright Law is authorized by SPIE subject to payment of copying fees. The Transactional Reporting Service base fee for this volume is $\$ 18.00$ per article (or portion thereof), which should be paid directly to the Copyright Clearance Center (CCC), 222 Rosewood Drive, Danvers, MA 01923. Payment may also be made electronically through CCC Online at copyright.com. Other copying for republication, resale, advertising or promotion, or any form of systematic or multiple reproduction of any material in this book is prohibited except with permission in writing from the publisher. The CCC fee code is 0277-786X/08/\$18.00.

Printed in the United States of America.

Publication of record for individual papers is online in the SPIE Digital Library.

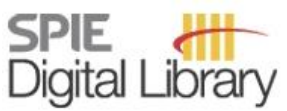

SPIEDigitalLibrary.org

Paper Numbering: Proceedings of SPIE follow an e-First publication model, with papers published first online and then in print and on CD-ROM. Papers are published as they are submitted and meet publication criteria. A unique, consistent, permanent citation identifier (CID) number is assigned to each article at the time of the first publication. Utilization of CIDs allows articles to be fully citable as soon they are published online, and connects the same identifier to all online, print, and electronic versions of the publication. SPIE uses a six-digit CID article numbering system in which:

- The first four digits correspond to the SPIE volume number.

- The last two digits indicate publication order within the volume using a Base 36 numbering system employing both numerals and letters. These two-number sets start with $00,01,02,03,04,05$, 06, 07, 08, 09, OA, OB ... 0Z, followed by 10-1Z, 20-2Z, etc.

The CID number appears on each page of the manuscript. The complete citation is used on the first page, and an abbreviated version on subsequent pages. Numbers in the index correspond to the last two digits of the six-digit CID number. 


\section{Contents}

HIGH-PERFORMANCE LASERS

699702 GaSb-based VECSEL exhibiting multiple-watt output power and high beam quality at a lasing wavelength of $\mathbf{2 . 2 5} \boldsymbol{\mu m}$ (Invited Paper) [6997-01]

B. Rösener, N. Schulz, M. Rattunde, C. Manz, K. Köhler, J. Wagner, Fraunhofer-Institut für Angewandte Festkörperphysik (Germany)

699703 The physics of catastrophic optical damage in high-power AIGalnP laser diodes [6997-02] M. Bou Sanayeh, OSRAM Opto Semiconductors GmbH (Germany) and Univ. DuisburgEssen (Germany); P. Brick, W. Schmid, B. Mayer, M. Müller, M. Reufer, K. Streubel, OSRAM Opto Semiconductors GmbH (Germany); M. Ziegler, J. W. Tomm, Max-Born-Institut (Germany); G. Bacher, Univ. Duisburg-Essen (Germany)

699705 Diffraction coupling of semiconductor tapered lasers in a very compact external Talbot cavity [6997-04] I. Hassiaoui, N. Michel, M. Lecomte, O. Parillaud, M. Calligaro, M. Krakowski, Alcatel-Thales III-V Lab. (France); R. McBride, Power Photonic (United Kingdom); G. Bourdet, Ecole Polytechnique (France); J.-P. Huignard, Thales Research \& Technology (France)

\section{SEMICONDUCTOR LASERS I}

699706 Applications of Gunn lasers [6997-05]

N. Balkan, S. H. Chung, Univ. of Essex (United Kingdom)

699707 Harmonic-resonance enhanced third-harmonic generation and Kerr-effect in mid-infrared quantum-cascade lasers [6997-06]

J. Bai, Univ. of Minnesota, Duluth (USA); D. S. Citrin, Georgia Institute of Technology (USA) and Unité Mixte Internationale, Georgia Tech-CNRS, Georgia Tech Lorraine (France)

699708 Spectral measurements and simulations of 405 nm (Al, In)GaN test laser structures grown on SiC and GaN substrate [6997-07]

T. Meyer, Univ. Regensburg (Germany) and Osram Opto Semiconductors GmbH (Germany); H. Braun, U. T. Schwarz, Univ. Regensburg (Germany); D. Queren, M. O. Schillgalies, S. Brüninghoff, A. Laubsch, U. Strauß, Osram Opto Semiconductors $\mathrm{GmbH}$ (Germany)

699709 Effects of detuned loading on the modulation performance of widely tunable MG-Y lasers [6997-08]

M. G. Chaciński, R. Schatz, Royal Institute of Technology KTH (Sweden); M. Isaksson, Syntune AB (Sweden); O. Kjebon, Royal Institute of Technology KTH (Sweden); Q. Wang, Acreo AB (Sweden) 
6997 OA A new simulation technique for DFB lasers [6997-09]

B. Heubeck, C. Pflaum, Univ. of Erlangen-Nuremberg (Germany)

COUPLED LASERS, CHAOS, AND SYNCHRONISATION

6997 OB Delay-coupled semiconductor lasers near locking: a bifurcation study [6997-10]

B. Krauskopf, Univ. of Bristol (United Kingdom); H. Erzgräber, Univ. of Exeter (United Kingdom)

6997 OC Fabrication and characterization of GalnNAs/GaAs semiconductor optical amplifiers [6997-11]

J. Pozo, Univ. of Bristol (United Kingdom) and Eindhoven Univ. of Technology (Netherlands);

N. Vogiatzis, O. Ansell, P. J. Heard, J. M. Rorison, Univ. of Bristol (United Kingdom);

P. Tuomisto, J. Konttinen, M. Saarinen, C. Peng, J. Viheriälä, T. Leinonen, M. Pessa, Tampere Univ. of Technology (Finland)

6997 OD Masking the time-delay of the chaotic output of an external-cavity laser [6997-12] D. Rontani, Supélec, LMOPS-CNRS (France), Georgia Tech-CNRS, Georgia Tech Lorraine (France), and Georgia Institute of Technology (USA); A. Locquet, Georgia Tech-CNRS, Georgia Tech Lorraine (France); M. Sciamanna, Supélec, LMOPS-CNRS (France) and Georgia Tech-CNRS, Georgia Tech Lorraine (France); D. S. Citrin, Georgia Tech-CNRS, Georgia Tech Lorraine (France) and Georgia Institute of Technology (USA)

6997 OE Time delay extraction in chaotic cryptosystems based on optoelectronic feedback with variable delay [6997-13]

S. Ortín, Univ. de Cantabria (Spain); M. Jacquot, CNRS-FEMTO-ST, Univ. de Franche-Comté (France); L. Pesquera, Univ. de Cantabria (Spain); M. Peil, L. Larger, CNRS-FEMTO-ST, Univ. de Franche-Comté (France)

6997 OF Synchronization on excitable pulses in optically injected semiconductor lasers [6997-14] O. Vaudel, N. Péraud, P. Besnard, CNRS Foton-Enssat (France)

\section{OPTICAL INJECTION AND DYNAMICS}

6997 0J All-optical frequency conversion using nonlinear dynamics of semiconductor lasers subject to external optical injection [6997-19]

S.-K. Hwang, National Cheng Kung Univ. (Taiwan); C.-Y. Lin, D.-H. Liang, National Chung Cheng Univ. (Taiwan)

6997 ON Optical bistability and flip-flop operation in DFB laser diodes injected with a CW signal [6997-23]

G. Morthier, K. Huybrechts, Ghent Univ. (Belgium)

699700 Bifurcation analysis of a multi-transverse-mode VCSEL [6997-24]

K. Green, Vrije Univ. (Netherlands); B. Krauskopf, Univ. of Bristol (United Kingdom)

6997 OP Asymmetric square-waves in mutually coupled semiconductor lasers [6997-25]

D. W. Sukow, Washington and Lee Univ. (USA); A. Gavrielides, Air Force Research Lab. (USA); T. Erneux, Univ. Libre de Bruxelles (Belgium); J. W. Davis, M. Z. Dube, S. Mabuza, R. Trimmer, Washington and Lee Univ. (USA) 
$69970 Q \quad 3.5$ W GalnNAs disk laser operating at 1220 nm (Invited Paper) [6997-26]

M. Guina, V.-M. Korpijärvi, J. Rautiainen, Tampere Univ. of Technology (Finland);

P. Tuomisto, EpiCrystals, Inc. (Finland); J. Puustinen, A. Härkönen, O. Okhotnikov, Tampere Univ. of Technology (Finland)

6997 OR Novel chirped multilayer quantum-dot lasers [6997-27]

G. Lin, C. Y. Chang, W. C. Tseng, C. P. Lee, National Chiao-Tung Univ. (Taiwan); K. F. Lin, R. Xuan, Industrial Technology Research Institute (Taiwan); J. Y. Chi, National Dong-Hwa Univ. (Taiwan)

6997 OS Difference in partition noise for $1.55 \mu \mathrm{m}$ quantum-dash and bulk structures [6997-28] J.-F. Hayau, J. Poëtte, V. Roncin, P. Besnard, FOTON-ENSSAT, CNRS (France); F. Lelarge, B. Rousseau, L. Le Govezigou, F. Pommereau, F. Poingt, O. Le Govezigou, A. Shen, G.-H. Duan, Alcatel-Thales III-V Lab. (France)

6997 OU A self-biased extremely shallow quantum-well SESAM with a low saturation fluence [6997-30]

E. A. Avrutin, Univ. of York (United Kingdom); B. S. Ryvkin, A.F. Ioffe Physico-Technical Institute (Russia) and Vrije Univ. Brussel (Belgium); K. Panajotov, Vrije Univ. Brussel (Belgium) and Institute of Solid State Physics (Bulgaria)

6997 OV Comprehensive RF-domain spectral- and time-domain analysis of passively mode-locked two-section quantum-dot lasers emitting at $1.3 \mu \mathrm{m}$ [6997-31]

S. Brever, L. Drzewietzki, W. Elsäßer, Darmstadt Univ. of Technology (Germany); M. Hopkinson, Univ. of Sheffield (United Kingdom); M. Calligaro, M. Krakowski, Alcatel Thales III-V Lab. (France)

VCSELS AND MICRO-OPTICS: JOINT SESSION WITH CONFERENCE 6992

6997 OW Photonic-crystal vertical-cavity surface-emitting lasers (Invited Paper) [6997-32]

D. F. Siriani, Univ. of Illinois at Urbana-Champaign (USA); P. O. Leisher, nLight Photonics

Corp. (USA); K. D. Choquette, Univ. of Illinois at Urbana-Champaign (USA)

6997 OX Microstructured photonic crystal for single-mode long wavelength VCSELs [6997-33]

R. Stevens, P. Gilet, A. Larrue, L. Grenovillet, N. Olivier, P. Grosse, K. Gilbert, B. Hladys,

B. Ben Bakir, CEA-LETI, MINATEC (France); J. Berggren, M. Hammar, Royal Institute of

Technology (Sweden); A. Chelnokov, CEA-LETI, MINATEC (France)

6997 OY Single mode $1.3 \mu \mathrm{m}$ InGaAs VCSELs for access network applications [6997-34]

P. Westbergh, E. Söderberg, J. S. Gustavsson, P. Modh, A. Larsson, Chalmers Univ. of Technology (Sweden); Z. Zhang, J. Berggren, M. Hammar, Royal Institute of Technology (Sweden)

$69970 Z$ Experimental study of transverse mode dynamics in vertical-cavity surface-emitting lasers under current modulation [6997-35]

A. Valle, Univ. de Cantabria (Spain); M. Arizaleta, Univ. Pública de Navarra (Spain);

K. Panajotov, Vrije Univ. Brussel (Belgium) and Institute of Solid State Physics (Bulgaria);

M. Sciamanna, Supélec, LMOPS-CNRS (France) 
699711 High power semiconductor disk laser with monolithically integrated pump lasers (Invited Paper) [6997-37]

W. Diehl, Osram Opto Semiconductors GmbH (Germany) and Philipps Univ. Marburg (Germany); T. Albrecht, P. Brick, M. Furitsch, S. Illek, S. Lutgen, I. Pietzonka, J. Luft, Osram Opto Semiconductors GmbH (Germany); W. Stolz, Philipps Univ. Marburg (Germany)

699712 Coherence radius and mode size of a broad-area vertical-cavity surface-emitting laser in the incoherent emission regime [6997-38]

G. Craggs, G. Verschaffelt, M. Peeters, Vrije Univ. Brussel (Belgium); S. K. Mandre, Darmstadt Univ. of Technology (Germany); I. Fischer, Vrije Univ. Brussel (Belgium)

699713 Excitation of a two-mode limit cycle dynamics on the route to polarization switching in a VCSEL subject orthogonal to optical injection [6997-39]

I. Gatare, Supélec, LMOPS-CNRS (France) and Vrije Univ. Brussel (Belgium); M. Sciamanna, Supélec, LMOPS-CNRS (France); M. Nizette, Univ. Libre de Bruxelles (Belgium); H. Thienpont, K. Panajotov, Vrije Univ. Brussel (Belgium)

699714 Unusual transverse-mode selectivity in some detuned VCSELs [6997-40]

R. P. Sarzała, M. Kuc, K. Gutowski, W. Nakwaski, Technical Univ. of Lódź (Poland)

\section{RING LASERS}

699716 High-speed integrated semiconductor micro-ring lasers with efficient off-axis parabolic reflectors [6997-41]

G. Verschaffelt, Vrije Univ. Brussel (Belgium); Z. Wang, Y. Shu, Univ. of Bristol (United Kingdom); G. Mezosi, Univ. of Glasgow (United Kingdom); J. Danckaert, Vrije Univ. Brussel (Belgium); M. Sorel, Univ. of Glasgow (United Kingdom); S. Yu, Univ. of Bristol (United Kingdom)

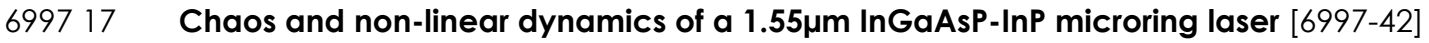
K. E. Chlouverakis, S. Mikroulis, D. Syvridis, Univ. of Athens (Greece)

699718 The dynamic behavior of a semiconductor ring laser [6997-43] G. Van der Sande, Univ. Illes Balears (Spain) and Vrije Univ. Brussel (Belgium); L. Gelens, P. Tassin, Vrije Univ. Brussel (Belgium); A. Scirè, Univ. Illes Balears (Spain); J. Danckaert, Vrije Univ. Brussel (Belgium)

\section{SEMICONDUCTOR LASERS III}

699719 Investigation on the linewidth enhancement factor of multiple longitudinal mode semiconductor lasers [6997-44]

A. Villafranca, Univ. of Zaragoza (Spain); G. Giuliani, S. Donati, Univ. di Pavia (Italy);

I. Garcés, Univ. of Zaragoza (Spain)

6997 IA Frequency stabilized high brightness tapered amplifier and laser modules [6997-45] G. Kochem, M. Haverkamp, K. Boucke, Fraunhofer Institute for Laser Technology (Germany) 
6997 1B Traveling wave modeling of semiconductor ring lasers [6997-46]

M. Radziunas, Weierstraß-Institut für Angewandte Analysis und Stochastik (Germany)

POSTER SESSION

6997 1D Third-order harmonic-expansion analysis of the Lorenz-Haken equations [6997-48]

S. Ayadi, Institut de Physique (Algeria); B. Meziane, Univ. d'Artois, UCCS Artois-CNRS

(France)

6997 IE Dynamic gain structuring during pulse build-up in self-pulsing lasers [6997-49]

B. Meziane, Univ. d'Artois, UCCS Artois-CNRS (France)

6997 IG Optimal laser diode operating mode with unstable operating temperature in turbulent atmosphere [6997-51]

L. Dordova, O. Wilfert, Brno Univ. of Technology (Czech Republic)

6997 1H Optimization of an avionic VCSEL-based optical link through large signal characterization [6997-52]

K. S. Ly, Airbus France S.A.S. (France) and ISAE/MOSE Group (France); A. Rissons, ISAE/MOSE Group (France); E. Gambardella, Airbus France S.A.S. (France); J.-C. Mollier, ISAE/MOSE Group (France)

699711 Compact illumination modules based on high-power VCSEL arrays [6997-53]

C. Gimkiewicz, M. Columbus, Ctr. Suisse d'Electronique et de Microtechnique SA (Switzerland); M. Moser, Bookham, Inc. (Switzerland)

$69971 \mathrm{~J}$ Polarization bistability in $\mathbf{1 . 5}$ micron wavelength single-mode vertical-cavity surfaceemitting lasers induced by orthogonal optical injection [6997-54]

A. Valle, M. Gómez-Molina, L. Pesquera, Univ. de Cantabria (Spain)

6997 1K Mode structure of a semiconductor laser with feedback from two external filters [6997-55] P. Słowiński, B. Krauskopf, Univ. of Bristol (United Kingdom); S. Wieczorek, Univ. of Exeter (United Kingdom)

6997 IL Bifurcations of composite-cavity modes in multi-stripe laser arrays [6997-56]

H. Erzgräber, S. Wieczorek, Univ. of Exeter (United Kingdom); B. Krauskopf, Univ. of Bristol (United Kingdom)

$69971 \mathrm{M}$ Oscillator-like resonance in the Maxwell-Bloch equations self-pulsing regime [6997-57] B. Meziane, Univ. d'Artois, UCCS Artois-CNRS (France)

6997 iN Modelling strategies for semiconductor ring lasers [6997-58]

A. Pérez S., Univ. Illes Balears (Spain); S. Fürst, Univ. of Glasgow (United Kingdom); A. Scirè,

J. Javaloyes, S. Balle, Univ. Illes Balears (Spain); M. Sorel, Univ. of Glasgow (United Kingdom)

699710 Nonlinear dynamics of a vertical-cavity surface-emitting laser subject to a repetitive optical pulse injection [6997-59]

Y.-S. Chang, F.-Y. Lin, National Tsing Hua Univ. (Taiwan) 
6997 IP Dynamical characteristics of a semiconductor laser injected by optical pulses with high repetition rate [6997-60]

Y.-S. Juan, F.-Y. Lin, National Tsing Hua Univ. (Taiwan)

$69971 Q \quad$ Noise properties of semiconductor ring lasers [6997-61]

A. Pérez S., R. Zambrini, A. Scirè, P. Colet, Univ. Illes Balears (Spain)

6997 IR Oblong-shaped VCSELs with pre-defined mode patterns [6997-62]

A.-S. Gadallah, A. Kroner, I. Kardosh, F. Rinaldi, R. Michalzik, Ulm Univ. (Germany)

6997 is Characterization of the chirp behavior of integrated laser modulators (ILM) by

measurements of its optical spectrum [6997-63]

I. Garcés, A. Villafranca, J. Lasobras, Univ. de Zaragoza (Spain)

6997 1T High power Al-free DFB laser diode for atomic clocks: narrow line-width and demonstration of saturation spectra of the cesium $D_{2}$ line [6997-64]

V. Ligeret, Alcatel Thales III-V Lab. (France) and Observatoire de Paris (France);

D. Holleville, Observatoire de Paris (France); M. Calligaro, M. Lecomte, Alcatel Thales III-V Lab. (France); S. Bansropun, Thales Research and Technology (France); O. Parillaud,

Alcatel Thales III-V Lab. (France); N. Dimarcq, Observatoire de Paris (France); M. Krakowski, Alcatel Thales III-V Lab. (France)

$69971 \mathrm{U}$ Measurement and simulation of the lateral mode profile of broad ridge $405 \mathrm{~nm}$ (Al,In)GaN laser diodes [6997-65]

H. Braun, D. Scholz, Univ. of Regensburg (Germany); T. Meyer, Univ. of Regensburg

(Germany) and Osram Opto Semiconductors GmbH (Germany); U. T. Schwarz, Univ. of

Regensburg (Germany); D. Queren, M. Schillgalies, S. Brüninghoff, A. Laubsch, U. Strauß,

Osram Opto Semiconductors GmbH (Germany)

6997 IV Carrier transport study in GalnNAs material using Monte-Carlo method [6997-67]

N. Vogiatzis, Y. N. Qiu, J. M. Rorison, Univ. of Bristol (United Kingdom)

6997 1W Optimization of the wall-plug efficiency of Al-free active region diode lasers at $975 \mathrm{~nm}$ [6997-68]

N. Michel, M. Lecomte, O. Parillaud, M. Calligaro, Alcatel-Thales III-V Lab. (France);

J. Nagle, Thales Research \& Technology (France); M. Krakowski, Alcatel-Thales III-V Lab. (France)

6997 1X Experimental investigation of relaxation oscillations resonance in mode-locked FabryPerot semiconductor lasers [6997-69]

V. Roncin, J. Poëtte, J.-F. Hayau, P. Besnard, J.-C. Simon, CNRS, Foton-Enssat (France);

F. Van Dijk, A. Shen, G.-H. Duan, Alcatel-Thales III-V Lab. (France)

6997 IY Frequency division multiplexed radio-over-fiber transmission using an optically injected laser diode [6997-70]

S.-C. Chan, City Univ. of Hong Kong (Hong Kong China)

699720 Broad-waveguide passively mode-locked laser diodes for stable short pulse generation [6997-72]

E. A. Avrutin, Univ. of York (United Kingdom); B. S. Ryvkin, E. L. Portnoi, A.F. loffe Physico-

Technical Institute (Russia) 
699721 Longitudinal multimode dynamics in a Fabry-Perot semiconductor microresonator [6997-73]

L. Columbo, L. Gil, Institut Non Linéaire de Nice, CNRS-UNSA (France)

699722 RIN spectra of a two-mode lasing two-section DFB laser for optical sensor application [6997-74]

H. Krause, J. H. Sonksen, J. Baumann, Univ. of Kassel (Germany); U. Troppenz, W. Rehbein, Fraunhofer-Institut für Nachrichtentechnik, Heinrich-Hertz-Institut (Germany); V. Viereck, H. H. Hillmer, Univ. of Kassel (Germany)

699723 Study and fabrication of buried oxide layers in GaAs/AIAs structures for confinement engineering in photonic devices [6997-75]

I. Suarez, M. Condé, G. Almuneau, L. Jalabert, P. Dubreuil, J. B. Doucet, L. Bouscayrol, C. Fontaine, LAAS-CNRS, Univ. de Toulouse (France)

699724 Time-domain response to ps optical pulse trigger of an all-optical flip-flop based on semiconductor ring laser [6997-76]

A. Trita, Univ. degli Studi di Pavia (Italy); S. Furst, G. Mezosi, M. Sorel, Univ. of Glasgow (United Kingdom); M. J. Latorre Vidal, J. YU, F. Bragheri, I. Cristiani, G. Giuliani, Univ. degli Studi di Pavia (Italy)

699725 Experimental analysis of the optical spectra of directionally bistable semiconductor ring lasers [6997-77]

M. J. Latorre Vidal, Univ. degli Studi di Pavia (Italy); S. Furst, G. Mezosi, Univ. of Glasgow (United Kingdom); M. Zanola, Univ. degli Studi di Pavia (Italy); M. Sorel, Univ. of Glasgow (United Kingdom); A. Perez, A. Scirè, S. Balle, Univ. Illes Balears (Spain); G. Giuliani, Univ. degli Studi di Pavia (Italy)

Author Index 
Downloaded From: https://www.spiedigitallibrary.org/conference-proceedings-of-spie on 25 Apr 2023

Terms of Use: https://www.spiedigitallibrary.org/terms-of-use 


\title{
Conference Committee
}

\author{
Symposium Chairs
}

Hugo Thienpont, Vrije Universiteit Brussel (Belgium)

Giancarlo C. Righini, Istituto di Fisica Applicata Nello Carrara (Italy)

Patrick P. Meyrueis, Université Louis Pasteur (France)

\section{Conference Chairs}

Krassimir P. Panajotov, Vrije Universiteit Brussel (Belgium) and Institute of Solid State Physics (Bulgaria)

Marc Sciamanna, Supélec (France)

Angel A. Valle, Universidad de Cantabria (Spain)

Rainer Michalzik, Universität Ulm (Germany)

Program Committee

Dieter Bimberg, Technische Universität Berlin (Germany)

Kent D. Choquette, University of Illinois at Urbana-Champaign (USA)

Shun Lien Chuang, University of Illinois at Urbana-Champaign (USA)

Harmen J. S. Dorren, Technische Universiteit Eindhoven (Netherlands)

Wolfgang E. Elsäßer, Technische Universität Darmstadt (Germany)

Athanasios T. Gavrielides, Air Force Research Laboratory (USA)

Claire Gmachl, Princeton University (USA)

Karlheinz H. Gulden, Bookham, Inc. (Switzerland)

Fritz Henneberger, Humboldt-Universität zu Berlin (Germany)

Ortwin G. Hess, University of Surrey (United Kingdom)

Anders G. Larsson, Chalmers Tekniska Högskola (Sweden)

Jia-Ming Liu, University of California, Los Angeles (USA)

Luigi A. Lugiato, Università degli Studi dell'Insubria (Italy)

Wlodzimierz Nakwaski, Politechnika Lódzka (Poland)

Junji Ohtsubo, Shizuoka University (Japan)

Dimitris Syvridis, University of Athens (Greece)

Atsushi Uchida, Takushoku University (Japan)

\section{Session Chairs}

1 High-Performance Lasers

Fritz Henneberger, Humboldt-Universität zu Berlin (Germany)

2 Semiconductor Lasers I

Wolfgang E. Elsäßer, Technische Universität Darmstadt (Germany) 
3 Coupled Lasers, Chaos, and Synchronisation

Angel A. Valle, Universidad de Cantabria (Spain)

4 Cavity Solitons

Giovanna Tissoni, INFM-CNR and CNISM, Università degli Studi dell'Insubria (Italy)

5 Optical Injection and Dynamics

Marc Sciamanna, Supèlec (France)

6 Semiconductor Lasers II

André Strittmatter, Technische Universität Berlin (Germany)

$7 \quad$ VCSELs and Micro-Optics: Joint Session with Conference 6992

Rainer Michalzik, Universität Ulm (Germany)

8 Vertical Cavity Lasers

Krassimir P. Panajotov, Vrije Universiteit Brussel (Belgium) and Institute of Solid State Physics (Bulgaria)

$9 \quad$ Ring Lasers

Athanasios T. Gavrielides, Air Force Research Laboratory (USA)

10 Semiconductor Lasers III

Ortwin G. Hess, University of Surrey (United Kingdom) 